\title{
FEMALE ATHLETE TRIAD
}

\section{A. PAige MorgenthAL, DC ${ }^{A}$}

${ }^{a}$ Professor and Chair, Department of Chiropractic Procedures, Southern California University of Health Sciences, Whittier, CA. Submit requests for reprints to: A. Paige Morgenthal, DC, Department of Chiropractic

Procedures, Los Angeles College of Chiropractic, Southern California University of Health Sciences, 16200 E. Amber Valley Drive, Whittier, CA 90609-1. Paper submitted May 20, 2002

\begin{abstract}
Objective: To describe the components of female athlete triad, the proposed mechanisms that may relate to its causation, and some screening and treatment options that may be used by the clinician who encounters this clinical entity in patients.
\end{abstract}

Data Sources: A qualitative review of the literature was performed.

Results: This article provides the clinician with current information relevant to understanding and recognizing female athlete triad in at-risk patients. The components of the triadamenorrhea, disordered eating, and osteoporosis-are interrelated in multifactorial etiology, pathogenesis, and consequences. Proposed causal factors include inadequate nutrition, menstrual status, training intensity and frequency, body size and composition, and psychological and physiological stress. There should be a high index of suspicion in all females athletes for the presence of and/or increased risk for female athlete triad. An increased awareness of the existence of the problem and its presenting signs and symptoms is the key to screening for this syndrome. The most efficacious treatment lies in its prevention.

Conclusions: Female athlete triad is an interrelated, multifactorial syndrome comprised of disordered eating, amenorrhea, and osteoporosis. It is a common entity among female athletes. Prevention, early detection, and early treatment are absolutely essential to maintain the athletes health into maturity and to prevent the serious consequences of this triad. The long-term effects of some triad components are still unknown. (J Chiropr Med 2002;1:97-106)

KEY INDEXING TERMS: Women's Health; Athletics; Osteoporosis; Anorexia

\section{INTRODUCTION}

The most serious health-related problems that threaten women who exercise are those which comprise the female athlete triad: amenorrhea, disordered eating, and osteoporosis. Female athlete triad was officially recognized as a syndrome and named in June 1992, when a panel of experts was convened by the American College of Sports Medicine to discuss an area of growing concern in sports medicine (1). Female athlete triad occurs not only in elite athletes but also in physically active women and girls who participate in a wide range of activities. These disorders may lead to significant morbidity and even a high rate of mortality (2). Each disorder is significant in and of itself, but when all three components of the triad are present, there is potential for a more serious impact on a womans performance and overall health (3). The components of the triad are interrelated in etiology, pathogenesis, and consequences. Its etiology is multifactorial. Proposed causal factors include inadequate nutrition, menstrual status, training intensity and frequency, body size and composition, and psychological and physiological stress (4).

\section{Amenorrhea}

The female reproductive system is highly sensitive to physiological stress. The reported range of reproductive abnormalities, including delayed menarche, luteal phase defects, oligomenorrhea, and primary and secondary amenorrhea, is $6 \%$ to $79 \%$ of women engaged in athletic activity; the prevalence of these irregularities varies with the sports and level of competition (5). The reported prevalence of amenorrhea itself in the athletic population is between $5 \%$ and $46 \%$, well above the prevalence of $2 \%$ to $5 \%$ found in the general population. Athletic amenorrhea has been observed in a wide variety of sports including long distance running, gymnastics, ballet dancing, cycling, swimming, rowing, Nordic skiing, and body building. The highest frequency of amenorrhea has been found in ballet dancers and runners $(6,7)$. Refer to Table 1 .

De Souza and Metzger (6) assert the wide variation in the prevalence rate of exercise-induced amenorrhea can be attributed to various definitions used by different investigators to define amenorrhea as well as the various survey techniques used to estimate prevalence. Amenorrhea is defined as 0 to 3 menstrual periods per year. Secondary amenorrhea is defined as the absence of at least three to six consecutive menstrual cycles in postmenarchal women. Female athletes who begin training before puberty may also experience delayed menarche, defined as not having menstrual periods by 16 years of age (primary amenorrhea) (8). Oligomenorrhea is defined as menstrual cycles greater than 36 days 
TABLE 1

INCIDENCE OF MENSTRUAL IRREGULARITIES (AMENORRHEA AND OLIGOMENORRHEA) IN DIFFERENT ATHLETIC DISCIPLINES

\begin{tabular}{|c|c|c|c|}
\hline SUBJECTS & STUDY & $\begin{array}{l}\text { NUMBER OF } \\
\text { SUBJECTS }\end{array}$ & $\begin{array}{l}\text { PERCENTAGE WITH } \\
\text { IRREGULARITIES }\end{array}$ \\
\hline GENERAL POPULATION & PATTERSON ET AL, 1973 & 1862 & 1.8 \\
\hline GENERAL POPULATION & SINGH, 1981 & 900 & 5.0 \\
\hline COLLEGIATE RUNNERS & SANBORN ET AL, 1982 & 237 & 25.7 \\
\hline CROSS-COUNTRY RUNNERS & WAKAT ET AL, 1982 & 41 & 5.0 \\
\hline CROSS-COUNTRY RUNNERS & SHANGOLD \& LEVINE, 1982 & 393 & 6.0 \\
\hline COLLEGIATE RUNNERS & FEICHT ET AL, 1978 & 127 & 24.0 \\
\hline BALLET DANCERS & COHEN ET AL, 1982 & 32 & 37.0 \\
\hline BALLET DANCERS & CALABRESE ET AL, 1983 & 34 & 44.0 \\
\hline BALLET DANCERS & ABRAHAM ET AL, 1982 & 29 & 38.0 \\
\hline BALLET DANCERS & BROOKS-GUNN ET AL, 1987 & 53 & 59.0 \\
\hline BALLET DANCERS & FEICHT ET AL, 1978 & 128 & $6.0-43.0$ \\
\hline BALLET DANCERS & GLASS ET AL, 1987 & 67 & 34.0 \\
\hline COLLEGIATE SWIMMERS & SANBORN ET AL, 1982 & 197 & 12.3 \\
\hline ELITE CYCLISTS & SANBORN ET AL, 1982 & 33 & 12.1 \\
\hline
\end{tabular}

[SOURCEs: De SOUZA ANd METZger, 1991 (6); WARREN AND PERLROTH, 2001 (7).]

in length or 4 to 9 menstrual periods per year. Women with long periods of amenorrhea, oligomenorrhea, or both, risk developing osteoporosis, as alterations in reproductive endocrine function coupled with their concomitant estrogen deficiency can all potentially reduce bone mass.

Unfortunately, many female athletes and their parents and coaches suffer from serious misconceptions about exercise-induced menstrual dysfunction. One of the most common misconceptions, according to Dueck, Manore, and Matt $(9,10)$, is that menstruation stops when body fat levels fall to an optimal level for a given sport. Consequently, many athletes and coaches believe that athletic amenorrhea signifies appropriate training volume and intensity, when it more than likely indicates that the athletes are most likely overtrained and, thus, performing at less than optimal levels. Typically, amenorrheic women present with multiple endocrine abnormalities, including low estrogen and progesterone, elevated cortisol, low thyroid hormones, high prolactin, fatigue, depression, and decreased performance. A profile of the athlete most likely to have exercise-associated amenorrhea has been determined $(2,11,12)$. (Refer to Table 2)

Exercise-induced amenorrhea is generally considered a subset of hypothalamic amenorrhea in which the gonadotropin releasing hormone $(\mathrm{GnRH})$ pulse generator appears to be affected (13). The apparent suppression of the normal pulsatile secretion of GnRH in women with exercise-induced amenorrhea subsequently leads to a concomitant loss of normal pulsatility of luteinizing hormone (LH) and follicle stimulating hormone (FSH) (14). As a consequence of subsequent ovarian hormone
TABLE 2

CHARACTERISTICS OF EXERCISE-ASSOCIATED AMENORRHEA

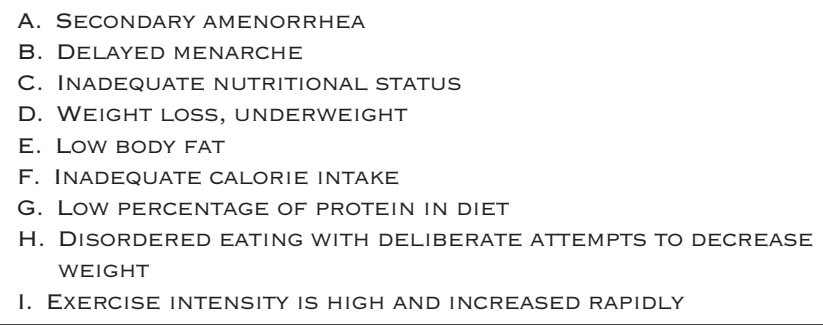

[SOURCES: NATTIV ET AL, 1994 (2); SNOW-HARTER, 1994 (11); MARSHALL, $1994(12)$.]

suppression, the levels of estrogen and progesterone fall. The characteristic hormonal profile of amenorrheic athletes includes elevated levels of glucocorticoids and depressed levels of estradiol and progesterone. These hormonal aberrations can have multiple adverse effects, including damage and inadequate repair of soft tissue, inhibition of bone formation and enhancement of bone resorption, inhibition of immune and thyroid function, and adverse effects on cardiovascular and renal function.

Burrows and Bird (15) described a number of variables that have been associated with the incidence of athletic amenorrhea, oligomenorrhea, and delayed menarche. Numerous studies have associated the prevalence of menstrual dysfunction with the age of the athletes, low body fat percentage, bodyweight changes, training intensity, age at menarche, intense training before menarche, prior menstrual irregularities, prior training histories, the endocrine conditioning model, the energy drain theory, pregnancy, and endogenous opioids (16- 
24). Several theories, stemming from study of the variables previously mentioned, have been proposed to account for the GnRH and LH pulsatility suppression that leads to menstrual dysfunction and irregularity.

The original hypothesis put forth by Frisch (25) suggested that a minimum of $17 \%$ body fat was necessary to bring about menarche in girls and a minimum of $22 \%$ body fat was necessary for women to sustain regularly cycling menstruation. Very soon thereafter, a number of studies provided data that showed that body composition does not vary significantly between eumenorrheic and amenorrheic athletes (26-36). Calabrese and coworkers found no difference in body fat between the 34 high-level amenorrheic and menstruating classical ballet dancers they studied (18). Sanborn and co-workers, in their study of 21 menstruating and 20 amenorrheic runners, found that both groups had the same percent body fat (34). Myerson and colleagues found that though both the eumenorrheic and amenorrheic runners they studied were lighter and leaner than sedentary controls, the runners percent body fat was the same (31).

Nonetheless, a number of other contemporaneous studies pointed to a pronounced association between training intensity, low body mass, and amenorrhea in athletic women. Sanborn and coworkers noted in their study of endurance swimmers, cyclists, and runners that amenorrhea was present in all three of the sports, but the prevalence was much higher among the runners $(25 \%$ vs. $12 \%)$ and was correlated with low body weight (3). Lutter and Cushman's study of runners published in the same year noted that there was a statistically significant trend toward fewer menstrual periods in runners with low body weight and high mileage (8). Carlberg and co-workers study of 42 female athletes, who participated in a number of different sports, concluded that menstrual dysfunction in athletes was associated with low body weight, comprised of smaller amounts of both fat and lean body mass (27). This conclusion was based on results that showed that athletes with oligo/amenorrhea were significantly lighter than athletes with regular menses, although both groups had body fat percentages well below the critical threshold hypothesized by Frisch and McArthur (25).

The exercise stress hypothesis is another theory put forth to explain the etiology of GnRH suppression (37$40)$. Some proposed stressors related to exercise are rapid loss of body weight, sudden onset of strenuous training or exercise, inadequate nutrition to meet energy requirements, and psychological and/or physi- ological stress. Simply stated, the exercise stress hypothesis maintains that the stress induced by intensive exercise, or its energy cost, activates the hypothalamicpituitary-adrenal axis such that high levels of cortisol secretion inhibit the hypothalamic-pituitary- ovarian axis and the release of GnRH. This disruption of GnRH pulsatility results in menstrual dysfunction. Bullen and coworkers (37), for example, reported a higher incidence of menstrual abnormalities in $85 \%$ of their test subjects following abrupt increases in intense physical activity that resulted in weight loss from negative energy balance. Abrupt increases in strenuous exercise without weight loss produced no menstrual changes. They concluded, vigorous exercise, particularly if compounded by weight loss, can reversibly disturb reproductive function in women. This study, as well as other experiments that have attempted to induce menstrual dysfunction through intense exercise, however, have shown that exercise coupled with caloric restriction effects LH suppression, but exercise alone has no effect on LH pulsatility.

These findings point to another hypothesis regarding suppressed GnRH pulsatility, the energy drain theory $(9,23,40)$. This theory maintains that an energy drain incurred by women whose energy expenditure exceeds dietary intake appears to be the primary factor effecting GnRH suppression in athletes engaged in sports emphasizing leanness. The proposition that low energy availability disrupts the GnRH pulse generator is based on widespread observation that women athletes consume less dietary energy than they would apparently need to sustain their high levels of activity. Dueck, Manore, and Matt $(9,10)$ assert that negative energy balance (energy drain) in athletic women is most likely related to three factors: high energy expenditure, low energy intake, and high psychological and physical stress.

Williams and coworkers (14) concluded from their study of four athletes that the neuroendocrine control of the reproductive axis is susceptible to short-term changes in energy availability. Moreover, strenuous training may not be a sufficient stimulus to disrupt reproductive hormone secretion unless accompanied by inadequate caloric intake. Their results suggested that a persistent combination of strenuous training and caloric insufficiency could result in sustained disruption of $\mathrm{LH}$ secretion and subsequently diminished gonadotropic support to the ovaries. In a sense, this study confirmed what Bullen and coworkers (37) had determined in their study a decade earlier. Most recently, Loucks, Verdun, and Heath (40), attempted to differentiate the independent effects of energy availability and exercise stress on LH pulsatility in nine regularly menstruating, 
nonobese, habitually sedentary women. They determined that four days of intense exercise have no disruptive effect on LH pulsatility apart from the impact of its energy cost on energy availability and that exercise energy expenditure disturbs LH pulsatility less than the equivalent amount of dietary energy restriction. In other words, exercise contributes to menstrual disturbances only when the energy expended is not adequately replenished through dietary intake. Athletic amenorrhea and other exercise-induced changes in menstrual cycling may be an energy-conserving strategy to protect more important biological and reproductive processes in female athletes with inadequate energy intake.

Perhaps most intriguing about the energy drain theory is how it links amenorrhea, one feature of female athlete triad, with another component of the triad, disordered eating. The combination of low body weight, variable nutrient or calorie intake, and exercise may act synergistically in the development of menstrual problems.

\section{Disordered Eating}

Disordered eating refers to a spectrum of abnormal patterns of eating. It includes behaviors of binging, purging, or both; food restriction; prolonged fasting; use of diet pills, diuretics, or laxatives; other abnormal eating behaviors; thought patterns such as preoccupation with food, dissatisfaction with ones body, fear of becoming fat, and a distorted body image. (Refer to Table 3). Anorexia nervosa and bulimia nervosa are at the extreme ends of the spectrum of disordered eating. Disordered eating behaviors can result in short and long term morbidity, decreased performance, amenorrhea, and even mortality (41-47).

The prevalence of disordered eating in young female athletes has been reported as $15 \%$ to $62 \%$. SundgotBorgen (48) maintains that symptoms of eating disor-

\section{TABLE 3}

\section{BEHAVIORAL SIGNS SUggeSTIVE OF DISORDERED EATING}

- PREoccupation With food and Weight

- REPEATEdly exPRESSED CONCERNS about being fat

- INCREASING CRITICISM OF ONE'S BODY

- frequently eats alone

- Use of LaXatives

- TRIPS to the bathroom duRINg or following meals

- Feet point toward the toilet in a bathroom stall

- CONTINUOUSLY DRINKS DIET SODA OR WATER

- Compulsive, excessive exercise

- COMPLAins of ALWAYS BEING COLD

[SOURCE: ROBERTS, 1995. (41)] ders are more prevalent among athletes than nonathletes; are more prevalent in female athletes than male athletes; and are more prevalent in sports in which leanness or a specific weight are considered important than in sports in which these factors are considered less important. The prevalence appears to be higher in adolescent and young women when the female athlete seems to be at significant risk for the development of the female athlete triad disorder (49). Greater prevalence has also been reported in persons from a higher socioeconomic background and with a family history of eating disorders.

Female athletes in sports in which low body weight and a lean physique are considered an advantage (e.g., gymnastics, figure skating, ballet dancing, swimming, diving, and distance running), especially athletes in elite or highly competitive levels, fit into a risk profile for female athlete triad. Athletes participating in individual sports appear to be at higher risk than athletes involved in team sports. Results of a recent study of collegiate athletes suggests that body weight concerns and dieting remain prevalent among female collegiate athletes, particularly those competing in aesthetics sports compared to those in endurance and team/anaerobic sports $(50,51)$. While the incidence of clinical eating disorders is similar among athletic groups, they concluded that athletes in aesthetic sports may be more at risk for subclinical eating disorders than those in endurance or team sports. Factors such as frequent weight cycling, pressure from important others to reduce weight, fear of puberty in female athletes, injury and overtraining, loss of a coach, fear of failing, and causal comments are among the factors considered to be associated with the development of clinical and subclinical eating disorders.

Increasing evidence supports the existence of subclinical or partial eating disorder syndromes among athletes who have weight control and body image problems, but lack the extreme psychopathological profiles of those with clinical eating disorders. Sundgot-Borgen has described anorexia athletica, a clinical variant of anorexia nervosa (47). In addition, Beals and Manore (26) have elaborated on the characteristics of subclinical eating disorders (SCED). Their study of 24 athletes with SCEDs compared to 24 control athletes determined that the characteristics most common in the female athlete with SCEDs include:

Preoccupation with food, energy intake (calories), and body weight;

Distorted body image and body weight and/or shape dissatisfaction; 
Undue influence of body weight and/or body shape on self-evaluation;

Intense fear of gaining weight, becoming fat, or feeling fat even though she may be at or slightly below $(\sim 5 \%)$ normal weight for height and/or body fat for sport;

Attempts to reduce body weight or maintain a lowered body weight and sport using one or a combination of pathogenic weight control methods, including severe restriction of energy intake, severe limitation of food choices and/or food groups, excessive exercise, fasting, self-induced vomiting, laxative and/or diuretic use;

Food intake governed by strict dietary rules and/or dietary boundaries (e.g., restriction of calories or fat grams to a specific amount, avoidance of specific foods or food groups, eating only certain times of the day, chronic avoidance of bad foods), accompanied by extreme feelings of guilt and self-hatred upon breaking a rule or surpassing a dietary boundary;

Absence of medical illness or affective disorder to explain energy restriction, weight loss and/or maintenance of low body weight or body fat percentage;

Menstrual dysfunction (26).

Inadequate calorie consumption may be the lowest common denominator in athletic amenorrhea and the female athlete triad. Studies measuring the energy intakes of female athletes, according to Beals and Manore (26), frequently report caloric intakes well below theoretical need or what would be predicted by energy expenditure. In general, studies comparing energy intakes of amenorrheic and eumenorrheic runners report that amenorrheic runners have lower energy intake (200 to $900 \mathrm{kcal}$ lower) than their eumenorrheic counterparts, despite similar body weights, body compositions, and training regimens. Kaiserauer and coworkers (22) noted that nutritional inadequacy would appear to separate the amenorrheic runners from the regularly menstruating runners. Myerson and coworkers (31) noted that the amenorrheic scored significantly higher than the eumenorrheic and sedentary on a scale of aberrant eating patterns and seem to have a less adequate diet than eumenorrheic runners. (Refer to Table 4)

The demands of training can outstrip calorie intake even among girls or women who dont restrict calories. Amenorrheic athletes frequently have lower energy, protein, and carbohydrate intakes than eumenorrheic athletes. These lowered intakes may also contribute to lower micronutrient intakes. Inadequate calorie intake may predispose these athletes to overtraining syndromechronic fatigue and decreasing performance despite adequate training levels. Disordered eating may also impair athletic performance and increase risk of injury. Decreased caloric intake and fluid and electrolyte imbalance may result in decreased endurance, strength, reaction time, speed, and ability to concentrate. Because the body initially adapts to these changes, a decrease in performance may not be seen for some time. However, food restriction and purging can result not only in menstrual dysfunction and potentially irreversible bone loss, but psychological and medical complications, including depression, fluid and electrolyte imbalance, and changes in the cardiovascular, endocrine, gastrointestinal, and thermoregulatory systems, some of which are potentially fatal.

Much attention has been focused on the psychological profile of girls and women with female athlete triad. Dueck, Manore, and Matt $(9,10)$ maintain exercise that is sufficient to disturb the normal hormonal balance in female athletes has been shown to be psychologically self-defeating, producing a mood state profile that is less desirable that that of inactive women. Cockerill and coworkers (52) found in their study of 20 women runners and 20 inactive women that the amenorrheic runners displayed a higher anger score than eumenorrheic runners or inactive women. They stated, training to the extent and intensity whereby an athlete becomes amenorrheic can have detrimental psychological consequences. It appears that the amenorrheic runners were

TABLE 4

SUMMARY OF STUDIES ON MEAN ENERGY INTAKES OF AMENORRHEIC AND EUMENORRHEIC RUNN

\begin{tabular}{|c|c|c|c|c|c|c|c|c|}
\hline \multirow[b]{2}{*}{ STUDY } & \multicolumn{3}{|c|}{ AMENORRHEIC } & \multicolumn{3}{|c|}{ EUMENORRHEIC } & \multirow{2}{*}{$\begin{array}{c}\text { KCAL } \\
\text { DIFFERENCE }\end{array}$} & \multirow[b]{2}{*}{$P$} \\
\hline & KCAL/DAY & KCAL/KG & MILES/WEEK & KCAL/DAY & $\mathrm{KCAL} / \mathrm{KG}$ & MILES/WEEK & & \\
\hline BAER \& TAPER, 1982 & 1991 & 44.0 & 40 & 1644 & 39.0 & 20 & +268 & NS \\
\hline DRINKWATER ET AL, 1984 & 1623 & 35.6 & 45 & 1965 & 41.0 & 25 & -342 & NS \\
\hline KAISERAUER ET AL, 1989 & 1582 & 36.0 & 39 & 2490 & 52.0 & 31 & -908 & $<.02$ \\
\hline MARCUS ET AL, 1985 & 1272 & 28.5 & 58 & 1715 & 36.0 & 58 & -443 & NS \\
\hline MYERSON ET AL, 1991 & 1731 & 39.5 & 53 & 1935 & 41.0 & 52 & -204 & NS \\
\hline NELSON ET AL, 1986 & 1730 & 38.3 & 35 & 2250 & 50.6 & 40 & -520 & $<.05$ \\
\hline WILMORE ET AL, 1991 & 1781 & 39.1 & $\sim 60$ & 1690 & 36.3 & $\sim 60$ & +91 & NS \\
\hline
\end{tabular}

[SOURCE: MODIFIED FROM BEALS AND MANORE, 1994 (22).] 
subjected to greater stresses than the other athletes and that this stress was both the cause and the outcome of athletic amenorrhea.

Sport and rigorous exercise may also play a pathogenic role in the onset and maintenance of eating disorders. Weight and Noakes (53) reported that the better athletes in their study of 125 female distance runners were more likely to exhibit the physical and psychological features of anorexia nervosa. Sundgot-Borgen assessed risk factors and triggers for eating disorders in female athletes (46). She found that prolonged periods of dieting, frequent weight fluctuations, a sudden increase in training volume, and traumatic events such as injury or loss of a coach were trigger factors associated with the onset of eating disorders. Davis and colleagues (43) examined seriously ill anorexic patients requiring intense hospital treatment. Their data indicated $78 \%$ of patients engaged in excessive exercise; $60 \%$ were competitive athletes prior to the onset of their disorder; $60 \%$ reported that sport or exercise predated dieting; and $75 \%$ claimed that physical activity levels steadily increased during the period when food intake and weight decreased the most. This study's authors concluded that overactivity should not be routinely viewed as a secondary symptom in anorexia nervosa equivalent to other behaviors. Instead, sport/exercise appears to be an integral part of the pathogenesis and progression of selfstarvation. There seems to be a powerful behavioral synergy and potentiation that occurs when starvation is combined with strenuous physical activity.

Many adverse consequences arise from the combination of disordered eating and athletic amenorrhea. The most serious health risk appears to be the adverse effects on bone health in girls and young women.

\section{Osteoporosis}

Although osteoporosis is most common among postmenopausal women, it occurs at any age when bone mass falls below a critical threshold. Osteoporosis is due to premature bone loss and inadequate bone formation; it results in low bone mass, microarchitectural deterioration, increased skeletal fragility, and an increased risk of fractures. Snow-Harter (11) asserts that exerciseassociated amenorrheic women are strong candidates for bone loss. She contends that osteoporosis is prevalent in female athletes of any age whose bone mass has fallen below the critical threshold (2.5 standard deviations below mean bone density for age-matched normally menstruating women).

The endocrine status of an amenorrheic female athlete shows a long-term estrogen-deficient state similar to that of postmenopausal women (54). Because estradiol is extremely important in facilitating calcium uptake into bone, amenorrhea and the associated hypoestrogenic state may predispose female athletes to osteopenia and premature osteoporosis. Increasing evidence has linked athletic amenorrhea to diminished bone mineral density (BMD). Early studies demonstrated that decreased BMD occurred in the axial spine, while more recent studies have demonstrated low BMD in both appendicular and axial skeletal sites.

Drinkwater and coworkers (54) concluded that the amenorrhea observed in female athletes may be accompanied by a decrease in mineral density of the lumbar vertebrae. They wrote that the average bone mineral density of the amenorrheic athletes (mean age of 24.9 years) was equivalent to that of women 51.2 years of age. The amount of physical activity reported by our amenorrheic athletes did not protect them from an apparent loss of vertebral bone. Their findings were similar to those of Cann and coworkers (5), who declared any factor that irreversibly decreases bone mass in women before the normal menopausal age of 50 years will predispose these women to the development of osteoporosis by bringing them closer to the fracture threshold earlier. To have the best chance of preventing further loss and possibly replacing some bone already lost, Cann and coworkers insisted that it is important to recognize women with premature bone loss as early as possible after the onset of amenorrhea, when bone turnover may be high from the metabolic stimulus of estrogen reduction (5).

Myburgh and colleagues (30) concluded that low BMD in athletes with amenorrhea is not limited to the axial skeleton but is also present in other regions including appendicular weight-bearing bones. They determined in this study that the lumbar spine BMD of amenorrheic subjects was $13 \%$ below predicted values for agematched normals and the deficit bone mineral at the femur was 13 to $19 \%$ below age-predicted values. Rencken and co-workers (33) further documented that amenorrheic athletes had significantly lower BMD $(p<.01)$ at the lumbar spine, femoral neck, trochanter, Ward triangle, intertrochanteric region, femoral shaft, and tibia.

Genetic predisposition has been found to be the strongest predictor of peak bone density, but menstrual history has also been found to be an important determinant. Studies have shown a direct relationship between both the severity and length of amenorrhea and menstrual cycle dysfunction and diminished BMD (55-62). Bone density increases in direct proportion to the num- 
ber of yearly menstrual cycles. The withdrawal of estrogens at any age is associated with bone loss that could lead to osteoporosis if prolonged. Myburgh and coworkers (30) found that among the anthropometric and historical variables tested, those related to menstrual history significantly predicted BMD. The best predictors of BMD were years of menstruation for lumbar spine; and years of amenorrhea for hip, femoral mid-shaft, and whole body. Prolonged weight-bearing exercise, they concluded, may not maintain bone mineral in these weight-bearing regions in the face of inadequate reproductive hormone production, as had been thought. Drinkwater, Bruemner, and Chestnut (54) also reported that vertebral BMD was significantly lower in athletes with a lifetime history of irregular menses, and that a linear relationship existed between menstrual irregularity and vertebral BMD. Mechanical loading on the skeleton from a variety of sports only has a beneficial effect on BMD when the athlete is estrogen-replete.

BMD is lowest in women with low levels of estrogen and progesterone and low body weight. In Rencken and coworkers study (33), duration of amenorrhea and body weight of amenorrheic athletes predicted BMD at the femoral neck, trochanter, intertrochanteric region, and tibia; weight alone predicted BMD at the femoral shaft and tibia. They asserted that since the length of the amenorrheic period is a significant factor in the generalized decrease in BMD, early identification and treatment may be necessary to protect young amenorrheic athletes from premature osteopenia-osteoporosis. The preferred treatment they suggested would be to encourage changes that lead to the resumption of normal menses, such as proper nutrition and weight gain.

Delayed menarche-associated with low cumulative levels of estrogen-can also negatively influence skeletal development, as nearly half of skeletal mass and $15 \%$ of final adult height are attained during adolescence. Bone mineral density has been shown to be inversely related to the age of menarche. Young athletes with delayed menarche have a lower bone age, as well as an increased risk of stress fractures. Athletes with delayed menarche may also enter adulthood with irreversible bone loss. Low estrogen levels have been linked to a reduction in bone mass. Girls with low reproductive hormones have substantial deficits in both cortical and trabecular bone. Estrogen deficiency appears to have a significant effect on bone mass even when adequate attention is given to other important influences on bone health, such as physical activity and calcium intake.

Failure to reach peak bone mass, premature loss of bone, and poor mineralization of bone all make the amenorrheic athlete more susceptible to stress fractures. Because of these factors, BMD may be decreased to the extent that stress fractures may occur with minimal loading impact of the bone. Athletes who develop oligomenorrhea also have reduced bone mineral density by as much as $20 \%$ compared with eumenorrheic athletes. Average bone density in the lumbar spine, femur, and wrist of amenorrheic athletes is anywhere from $9 \%$ to $31 \%$ less than the bone density of eumenorrheic athletes and sedentary controls.

Several studies have reported that amenorrheic athletes are at increased risk for stress fractures when compared with eumenorrheic athletes (63-66). The majority of these fractures occur in the lower extremities where overload is most likely to occur in weight-bearing activities. In two studies of ballet dancers, the incidence of stress fractures was reported as $31 \%$ and $45 \%$ respectively. In a study of distance runners, roentgenographically documented overt fractures occurred in $24 \%$ of amenorrheic women, compared with only $9 \%$ of women who were eumenorrheic. Athletes using oral contraceptives, in two studies had fewer stress fractures than nonusers did; the likelihood of injury was inversely correlated with contraceptive use and speaks to the protective properties of exogenous hormones on bone. However, another study showed no such association.

Certain studies suggest that premature osteoporosis may occur in the young female athlete and may be irreversible even with calcium supplementation. Drinkwater and coworkers initially found that exercise-induced amenorrheic BMD deficit was partially reversible during the first year following resumption of menses in the athletes they studied (54). However, two subsequent reports suggested that some young women may not be able to completely replace vertebral bone lost as a result of extended interruptions in the normal menstrual cycle $(28,29)$. Thus, menstrual history, as well as current menstrual status, has a significant affect on vertebral density.

\section{Screening and treatment options for Female Athlete Triad}

Nattiv and coworkers (2) maintain that the treatment of female athlete triad lies in its prevention. They also believe there should be a high index of suspicion in all female athletes for the presence of and/or increased risk for female athlete triad. An increased awareness of the existence of the problem and of the presenting signs and symptoms is the key to screening for female athlete triad. (Refer to Table 5.) The preparticipation physical 
TABLE 5

SigNS AND SYMPTOMS OF FEMALE ATHLETE TRIAD

$\checkmark$ Fatigue

- ANEMIa

- depression

- stress fracture

- Decreased ability to concentrate

- cold intolerance

- hypothermia

- Cold and discolored hands and feet

- enlargement of the parotid glands

- Sore throat

- Callused knuckles from pressure against teeth during INDUCED VOMITING

- EROsion of dental enAmel from fREquent Vomiting

- Abdominal pain and bloating

- Constipation

- DRY SKIN

- Face and extremity edema

$\checkmark$ Lightheadedness

- bradycardia

- Changes in orthostatic blood pressure

- Chest pain

- lanugo

- Carotenemia

[SOURCE: ROBERTS, 1995 (41).]

examination may be the ideal opportunity to screen the athlete for disordered eating, amenorrhea, or other patterns of menstrual irregularity, as well as a history of, or potential for, stress fractures. Life stressors, depressive symptoms, dissatisfaction with weight or body shape, training intensity, and other lifestyle behaviors also may be addressed at this time. The athlete will not usually bring such personal issues to the attention of the physician unless specifically asked.

Because disordered eating and inadequate energy intake are such integral factors in causation of the triad, nutritional education and counseling are a necessary part of preventing female athlete triad. To protect against osteoporosis, emphasis should be placed on the accumulation of high levels of bone mass through diet and exercise in the premenopausal stage, rather than treatment after menopause, or after bone loss has occurred. Maintaining normal menstruation, adequate caloric intake, and normal body weight are critical in girls and young women athletes so that they can accumulate bone mass. An individuals peak bone mass is the amount of bone each person has at the end of growth which occurs at some point between the 2nd and 3rd decades, usually by age 35. Women exhibiting higher levels of peak bone mass will experience similar agerelated bone loss as women with lower values but will be well above a critical fracture threshold because they had higher initial values. The greater the bone mass at maturity, the more a woman can afford to lose.

Restoring regularly cycling menstruation is also critical to the prevention of further bone loss in the amenor- rheic athlete. Insuring adequate daily caloric intake and a slight reduction in exercise intensity may be all that is necessary for the amenorrheic athlete to resume menses. In their studies, the athlete test subjects ingested a daily sports supplement shake for an additional $350 \mathrm{kcal}$ and refrained from exercise one day each week (13). This simple regimen alone was enough to restore most of the athletes to a positive energy balance, such that normal menses returned in 4 of 5 athletes. They concluded, however, that this is only effective when the athlete is not encumbered by many disordered eating characteristics and recognizes that weight loss or low body mass is hindering her performance.

In a recent pilot study, Sundgot-Borgen examined the effect of cognitive-behavioral therapy (CBT) versus the effect of nutritional counseling on 44 elite bulimic athletes (48). CBT appeared more effective than nutritional counseling in the reducing pursuit of thinness, frequency of binging, purging, and laxative abuse. Further study may provide additional support to her conclusion that CBT is important in the treatment of bulimic athletes.

Manore maintains that if a female athlete restricts her energy intake to less than $1800 \mathrm{kcal}$ per day, it is almost impossible to get adequate amounts of macro- and micronutrients to maintain good health and an intense training program (44). She suggests that any athlete who complains of fatigue, frequent injuries, irritability, or poor athletic performance should try increasing her daily caloric intake by 200 to 300 calories. Moreover, athletes should insure that they are in a positive energy balance before beginning any physical activity for the day. Manore provides some guidelines to help physicians and coaches determine whether a female athlete is consuming enough calories. If the female athletes energy intake is insufficient, she may experience the following symptoms:

- The female athlete may be hungry, irritable, and have difficulty concentrating before or during her exercise routine. Sometimes, she may even get shaky or lightheaded.

- An adolescent female athlete fails to grow.

- The female athlete has stopped menstruating or is having irregular periods throughout the year, especially during periods of intense exercise training.

- The female athlete is losing weight, especially during periods of intense exercise training (44).

Snow-Harter (11) also recommends a decrease in training intensity and volume and an increase in total calories and daily calcium intake (1200 to $1500 \mathrm{mg} /$ day) for

\section{4}


athletes experiencing loss of menstrual periods accompanied by low bone mass. A program of resistance training designed to increase both muscle strength and mass may improve the skeletal profile of amenorrheic and oligomenorrheic athletes as well as protect against soft tissue injuries. Finally, some suggest estrogen replacement therapy may be indicated for women, especially those who are not willing to make changes in their exercise or dietary patterns (67). Although it was generally accepted initially that the doses prescribed to postmenopausal women would be adequate for reducing bone loss among amenorrheic athletes, more recent data does not support this supposition. Amenorrheic athletes using hormone replacement therapy in doses used for menopausal women have shown maintenance of BMD, but no gains.

\section{CONCLUSION}

Female athlete triad is an interrelated syndrome comprised of disordered eating, amenorrhea, and osteoporosis. It is a common entity among female athletes. Although much has been done to raise the awareness of athletes, coaches, parents, and health-care providers about this condition and its serious consequences, more still needs to be done. Prevention, early detection, and early treatment are absolutely essential to maintain the athletes health into maturity. Averting serious sequelae of the triad is most important. However, the long-term effects of some of the triad components, for example, disordered eating and long-term dietary restriction, are still unknown.

Another question looms large on the horizon. Since Drinkwater and coworkers determined that amenorrheic athletes had the bone density of women thirty years their senior (28), many striking similarities have been seen with postmenopausal changes in bone and those seen in amenorrheic athletes. The common denominator in both populations is estrogen deficiency. There has been much speculation that another estrogen-related effect observed in postmenopausal women, cardiovascular disease, might also be found in amenorrheic athletes with female athlete triad (68). To date, it has not yet been determined definitively whether the hypoestrogenic state associated with athletic amenorrhea may predispose an individual to cardiovascular disease. Some studies, however, have shown adverse changes in lipid and lipoprotein profiles associated with menstrual dysfunction that may increase the risk for premature atherogenesis in amenorrheic athletes. Most recently, Zeni and co-workers (36), in a study of 10 amenorrheic, 11 oligomenorrheic, and 10 eumenorrheic runners, found evidence of premature cardiovascular disease, as measured by altered endothelial cell function and decreased capacity for arterial vasodilation in response to nitric oxide, in the amenorrheic runners. These changes are similar to those usually found in arteries of postmenopausal women. Surely the alarming findings in this preliminary study will spur further inquiry that may lead to the expansion of female athlete triad to a tetrad of interrelated disorders linked to female athleticism.

\section{REFERENCES}

1. Otis CL, Drinkwater B. American College of Sports Medicine Position Stand: The female athlete triad. Med Sci Sports Exercise 1997;29(5): i-ix

2. Nattiv A, Agostini R et al. The female athlete triad: the interrelatedness of disordered eating, amenorrhea, and osteoporosis. Clin Sports Med 1994; 13: 405-417

3. Sanborn CF, Horea $M$ et al. Disordered eating and the female athlete triad. Clin Sports Med 2000;19(2): 199-213

4. Yeager KK, R Agostini et al. The female athlete triad: disordered eating, amenorrhea, osteoporosis. Med Sci Sports Exercise 1993;25: 775-777

5. Cann CE, Martin MC et al.. Decreased spinal mineral content in amenorrheic women. JAMA 1984;251(5): 626-629

6. De Souza MJ, Metzger DA. Reproductive dysfunction in amenorrheic athletes and anorexic patients: a review. Med Sci Sports Exercise 1991;23(9): 995-1007

7. Warren MP, Perlroth NE. The effects of intense exercise on the female reproductive system. J Endocrinol 2001;170: 3-11

8. Lutter JM, Cushma S. Menstrual patterns in female runners. Physician Sports Med 1982;10(9): 60-72

9. Dueck CA, Manore MM. Role of energy balance in athletic menstrual dysfunction. Int J Sport Nutr 1996;6: 165-190

10. Dueck CA, Matt KS et al. Treatment of athletic amenorrhea with a diet and training intervention program. Int J Sport Nutr 1996;6: 24-40

11. Snow-Harter C. Bone health and prevention of osteoporosis in active and athletic women. Clin Sports Med 1994;13: 389-403

12. Marshall LA. Clinical evaluation of amenorrhea in active and athletic women. Clin Sports Med 1994;13: 371-38

13. Bonen A, Keizer HA. Athletic menstrual cycle irregularity: endocrine response to exercise and training. Physician Sports Med 1984;12(8): 78-94

14. Williams NL, Young JC et al. Strenuous exercise with caloric restriction: effect on luteinizing hormone secretion. Med Sci Sports Exercise 1995;27(10): 1390-1398

15. Burrows M, Bird S. The physiology of the highly trained female endurance runner. Sports Med 2000;30(4):281-300

16. Beals KA, Manore MM.The prevalence and consequences of subclinical eating disorders in female athletes. Int J Sport Nutrition 1994;4: 175-195

17. Brownell KD, Nelson Steen S et al.. Weight regulation practices in athletes: analysis of metabolic and health effects. Med Sci Sports Exercise 1987;19: 546-556

18. Calabrese LH, Kirkendall DT et al.. Menstrual abnormalities, nutritional patterns, and body composition in female classical ballet dancers. Physician Sports Med 1983;11(2): 86-97

19. Cumming DC. Exercise-associated amenorrhea, low bone density, and estrogen replacement therapy. Arch Internal Med 1996;156: 2193-2195

20. Harber VJ. Menstrual dysfunction in athletes: and energetic challenge. Exercise Sport Sci Rev 2000;28(1): 19-23

21. Highet R. Athletic amenorrhoea: an update on aetiology, complications, and management. Sports Med 1989;7: 82-108

22. Kaiserauer S, Snyder AC et al. Nutritional, physiological, and menstrual status of distance runners. Med Sci Sports Exercise 1989;21(2): 120-125

23. Kopp-Woodroffe SA, Manore MM et al. Energy and nutrient status of amenorrheic athletes participating in a diet and exercise training intervention programs. Int J Sport Nutr 1999;9: 70-88

24. Lloyd T, Triantafyllou SJ et al. Women athletes with menstrual irregularity have increased musculoskeletal injuries. Med Sci Sports Exercise $1986 ; 18(4): 374-379$ 
25. Frisch RE, Wyshak G et al. Delayed menarche and amenorrhea in ballet dancers. New England J Med 1980;03(1): 17-19

26. Beals KA, Manore MM. Body weight concerns and dieting practices of female collegiate athletes. J Am Diet Assoc 1998: A-13

27. Carlberg KA, Buckman MT et al. Body composition of oligo/amenorrheic athletes. Med Sci Sports Exercise 1983;15(3): 215-217

28. Drinkwater BL, Nilson $\mathrm{K}$ et al. Bone mineral content of amenorrheic and eumenorrheic athletes. New England J Med 1984;311: 277-281

29. Drinkwater BL, Nilson $\mathrm{K}$ et al. Bone mineral density after resumption of menses in amenorrheic athletes. JAMA 1986;256: 380-382

30. Myburgh KH, Bachrach LA et al. Low bone mineral density at axial and appendicular sites in amenorrheic athletes Medicine and Science in Sports and Exercise 1993;25(11): 1197-1202

31. Myerson M, Gutin B et al. Resting metabolic rate and energy balance in amenorrheic and eumenorrheic runners. Med Sci Sports Exercise $1991 ; 23(1): 15-22$

32. Nelson ME, Fisher EC et al. Diet and bone status in amenorrheic runners. Am J Clin Nutr 1986;43: 910-916

33. Rencken ML, Chestnut III CH et al. Bone density at multiples skeletal sites in amenorrheic athletes. JAMA 1996;276(3): 238-240

34. Sanborn CF, Albrecht BH et al. Athletic amenorrhea: lack of association with body fat. Med Sci Sports Exercise 1987;19(3): 207-212

35. Wilmore $\mathrm{JH}$, Wambsgans $\mathrm{KC}$ et al. Is there energy conservation in amenorrheic compared with eumenorrheic distance runners? J Applied Physiol 1992;72(1): 15-22

36. Zeni A. (2001). Is there an association between athletic amenorrhea and early cardiovascular disease? Baltimore, MD:American College of Sports Medicine, as yet unpublished

37. Bullen BA, Skrinar GS et al.. Induction of menstrual disorders by strenuous exercise in untrained women. New England J Med 1985:312: 1349-1353

38. Drinkwater B. Exercise and bones: Lessons learned from female athletes. Am J Sports Med 1996;24(6): S-33-S-35

39. Loucks AB. Effects of exercise training on the menstrual cycle: existence and mechanisms. Med Sci Sports Exercise 1990;22(3): 275-280

40. Loucks $A B$, Verdun $M$ et al. Low energy availability, not stress of exercise, alters LH pulsatility in exercising women. J Applied Physiol 1998;84(1): $37-46$

41. Roberts WO. Primary amenorrhea and persistent stress fracture: a practical clinical approach. Physician Sports Med 1995;23: 33-42

42. Beals KA, Manore MM. Behavioral, psychological, and physical characteristics of female athletes with subclinical eating disorders. Int J Sport Nutr Exercise Metabolism 2000;10: 128-143

43. Davis $\mathrm{CH}$, Kennedy D et al. The role of physical activity in the development and maintenance of eating disorders. Psychiatric Medicine 1994;24: 957-967

44. Manore MM. Nutritional needs of the female athlete. Clin Sports Med 1999;18(3): 549-563

45. Shangold M, Rebar RW et al. Evaluation and management of menstrual dysfunction in athletes. JAMA 1990;263: 1665-1669

46. Sundgot-Borgen J. Risk and trigger factors for the development of eating disorders in female elite athletes. Med Sci Sports Exercise 1994;26(4): $414-419$
47. Sundgot-Borgen J. Eating disorders among male and female athletes. Br J Sports Med 1990;33: 434

48. Sundgot-Borgen J. The long-term effect of CBT and nutritional counseling in treating bulimic elite athletes. Med Sci Sports Exercise 2001;33(5 supplement): S97

49. Benson JE, Engelbert-Fenton KE et al.. Nutritional aspects of amenorrhea in the female athlete triad. Int J Sport Nutrition 1996;6: 134-145

50. Sanborn CF, Martin BJ et al. Is athletic amenorrhea specific to runners? Am J Obstetrics Gynecol 1982;143: 859-861

51. Warren MP. Health issues for women athletes: exercise-induced amenorrhea. J Clin Endocrinol Metabolism 1999;84(6):1892-1896

52. Cockerill IM, Nevill AM et al. Mood, mileage, and the menstrual cycle. Br J Sports Med 1992;26(3): 145-150

53. Weight LM, Noakes TD. Is running an analog of anorexia?: a survey of the incidence of eating disorders in female distance runners. Med Sci Sports Exercise 1987;19(3): 213-217

54. Drinkwater B, Breumner B et al.. Menstrual history as a determinant of current bone density in young athletes. JAMA 1990;263: 545-548

55. Beim G, Stone DA. Sports medicine issues in the female athlete. Orthop Clin North Am 1995;26: 443-451

56. Benson JE, Geiger CJ et al.. Relationship between nutrient intake, body mass index, menstrual function, and ballet injury. J Am Dietetic Assoc 1989;89(1): 58-63

57. Cann CE, Cavanaugh DJ et al. Menstrual history is the primary determinant of trabecular bone density in women runners. Med Science Sports Exercise 1998;20(Supplement 2): S59

58. Kadel NJ, Teitz CC et al. Stress fractures in ballet dancers. Am J Sports Med 1992;20(4): 445-449

59. Myburgh KH, Hutchins J et al. Low bone density is an etiologic factor for stress fractures in athletes. Ann Internal Med 1990;113: 754-759

60. Voss L, Fadale P et al. Osteoporosis in young, athletic women. J Musculoskeletal Med 1996;13: 15-22

61. Wilson JH, Wolman RL. Osteoporosis and fracture complications in an amenorrhoeic athlete. Br J Rheumatol 1994;33: 480-481

62. Zanker C, Swaine IL. Relation between bone turnover, oestradiol, and energy balance in women distance runners. Br J Sports Medicine 1998;32: 167-171

63. Bennell KL, Malcolm SA. Risk factors for stress fractures in track and field athletes: a 12-month prospecitive study. Am J Sports Med 1996;24: $810-818$

64. Warren M, Brooks-Gunn PJ et al. Scoliosis and fractures in young ballet dancers: relation to delayed menarche and secondary amenorrhea. New England J Med 1999:314: 1348-1353

65. Thein LA, Thein JM. The female athlete. J Sports Phy Ther 1996;23: 134-148

66. Van De Loo DA, Johnson MD. The young female athlete. Clin Sports Med 1994;13: 687-707

67. Bennell KL, White SA. The oral contraceptive pill: a revolution for sportswomen? Br J Sports Med 1999;33: 231-23

68. Marcus R, Cann CA et al. Menstrual function and bone mass in elite women distance runners. Ann Internal Med 1985;102: 158-163 